\title{
Avaliação da reforma curricular do curso de odontologia da Universidade Federal de Santa Catarina
}

\author{
Gabriella Machado Vieira*, Graziela de Luca Canto**
}

\author{
* Aluna do curso de Graduação em Odontologia da Universidade \\ Federal de Santa Catarina \\ ** Professora Doutora do curso de Graduação em Odontologia da \\ Universidade Federal de Santa Catarina
}

\section{RESUMO}

O curso de Odontologia da Universidade Federal de Santa Catarina passou recentemente por alterações curriculares embasadas nas Diretrizes Curriculares Nacionais dos cursos de graduação em Odontologia. A alteração do projeto pedagógico foi realizada em 2006, em concordância com a Resolução no 3/02 CNE/CES, de 19 de fevereiro de 2002, que instituiu as Diretrizes Curriculares Nacionais do Curso de Graduação em Odontologia. O novo currículo entrou em vigor no primeiro semestre de 2007. Objetivando avaliar a reforma curricular a partir da comparação entre os conhecimentos de diagnóstico dos alunos do antigo e dos alunos do novo currículo, suas opiniões sobre o curso e suas aptidões profissionais foi aplicado um questionário contendo 20 questões sobre diagnóstico em Odontologia e 4 questões relacionadas ao curso de graduação e sua aptidão profissional. O estudo contou com a participação de 90 estudantes que formaram dois grupos, o grupo I formado pelos alunos do currículo antigo e o grupo II, formado pelos alunos do currículo novo. Após a aplicação dos questionários os dados foram calculados estatisticamente no programa epiData e digitados no Excel. Os resultados mostraram que as diferenças entre os conhecimentos de diagnóstico não foram estatisticamente significativas entre os grupos I e II, em relação ao ponto forte do curso houve diferença estatística entre os dois grupos, no grupo I predominou o aprendizado em modalidades de tratamento enquanto no grupo II o aprendizado em diagnóstico integral. Em relação ao local onde preferem atuar ambos os grupos responderam em sua maioria nos serviços públicos de saúde associado a clínica privada, em relação às áreas de atuação tanto o grupo I como o grupo II optou em sua maioria pelas disciplinas clínicas tendo uma prevalência relevante no grupo II a Saúde Coletiva.

\section{DESCRITORES}

Diagnóstico. Interdisciplinaridade. Odontologia. Currículo. Diretrizes Curriculares.

$\Delta$ regulamentação do exercício profissional da Odontologia data de 14 de maio de 1856, com o Decreto $\mathrm{n}^{\circ} 1.764$. O ensino formal só teve início com o Decreto n 7.247 de 19 de março de 1879, que estabeleceu o curso de "Cirurgia-dentária", anexo a faculdades de medicina (Abeno, 2010).

No Brasil, o ensino da Odontologia inspirou-se no modelo de ensino adotado nos Estados Unidos, que utiliza uma abordagem diferente do tradicionalismo europeu, no qual a Odontologia estava incorporada ao ensino da Medicina, destacando-se como especialidade médica. O modelo adotado pelos americanos originou um novo formato de ensino pelo direcionamento das disciplinas básicas às exigências e necessidades diretas da Odontologia (Rosa; Madeira, 1982).

No estado de Santa Catarina a primeira tentativa da fundação de uma faculdade de Odontologia data de 1909 quando foi projetada a Faculdade Livre de Farmácia, Odontologia e Obstetrícia; este projeto jamais saiu do papel. Somente no ano de 1917 foi fundado o Instituto Polytéchnico, e o curso de Odontologia fazia parte dos cursos desta instituição juntamente com Farmácia e Agrimensura; o Instituto passou por diversas crises e encerrou suas atividades no ano de 1932. De 1932 a 1946 o estado não contou com nenhum curso de Odontologia, neste ultimo ano foi inaugurada a Faculdade de Farmácia e Odontologia, uma instituição particular que conta- 
va basicamente com a mensalidade dos alunos, passou por diversas crises e culminou na sua inclusão na recém criada Universidade Federal de Santa Catarina em 1960 (Rosa; Madeira, 1982).

A federalização permitiu um avanço no ensino, trazendo modernidade e desenvolvimento situando-se na linha da frente no ensino odontológico brasileiro. Além de avanço, modernidade e desenvolvimento diversas reformas na organização curricular fizeram parte da historia do curso de Odontologia da UFSC (Rosa; Madeira, 1982).

Em 2006 o projeto pedagógico da organização curricular do curso de Graduação em Odontologia da Universidade Federal de Santa Catarina foi novamente reorientado, desta vez em concordância com a Resolução no 3/02 CNE/CES, de 19 de fevereiro de 2002, que instituiu as Novas Diretrizes Curriculares Nacionais para Cursos de Graduação em Odontologia, objetivando formar um cirurgião-dentista generalista, voltado para a promoção de saúde, que tenha autonomia e liderança, saiba trabalhar em equipe, atuando em todos os níveis de atenção a saúde, empregando as práticas da integralidade e interdisciplinaridade com educação e aprendizado constante (Amante, 2006).

A alteração curricular foi embasada nas premissas de diversos autores.

De acordo com Costa (2007), as novas exigências do mercado de trabalho almejam um profissional generalista e de formação mais ampla, preparado para extrapolar o espaço da clínica e propor um diagnóstico sobre o coletivo e suas intervenções. Para que isso aconteça Badan et al. (2010) dizem que o profissional de saúde moderno deverá pensar e atuar em seu meio enxergando-o de forma mais crítica e reflexiva considerando o contexto onde estão inseridos profissional e paciente. Todas essas transformações exigem alterações no protocolo de atendimento ao paciente. Vivencia-se uma época de valorização do ser humano, que preconiza uma melhor relação profissional-paciente (Amante, 2006).

Diante disto as Diretrizes Curriculares Nacionais dos cursos de Odontologia objetivam que o aprendizado dos futuros profissionais seja com autonomia e discernimento para garantir a integralidade da atenção e a qualidade e humanização do atendimento prestado aos indivíduos, famílias e comunidades (Brasil, 2002).

Em um curso da área da saúde, observa-se a relação de integração entre os cursos afins, no qual o paciente é visto como um todo e, não simplesmente, como uma parte (Gondim, 2002). Portanto quanto mais praticada a interdisciplinaridade maior a possibilidade de estabelecimento da integralidade nas práticas em saúde (Feuerwerker, 2003).

Dessa forma espera-se formar profissionais críticos, aptos a desenvolver ações de promoção, prevenção, proteção e reabilitação da saúde, capazes de trabalhar em equipe e de levar em conta a realidade epidemiológica e social para, embasados nesses princípios, prestar uma atenção à saúde mais humana $\mathrm{e}$ de qualidade (Costa; Araújo, 2011).

Com base nos dados citados anteriormente o presente estudo trás como principal objetivo avaliar a reforma curricular do curso de Odontologia da Universidade Federal de Santa Catarina, com ênfase nos conhecimentos de diagnóstico de alunos do antigo e de alunos do novo currículo, a opinião dos alunos sobre o ponto forte do ensino no curso de Odontologia UFSC e as aptidões dos estudantes como futuros profissionais.

\section{METODOLOGIA}

O projeto desta pesquisa foi encaminhado ao Comitê de Ética em Pesquisa com Seres Humanos da Universidade Federal de Santa Catarina, o qual foi julgado e aprovado de acordo com o parecer de $\mathrm{n}^{\circ}$ 0584/GR/99. De acordo com o projeto aprovado, os estudantes foram convidados a participar da pesquisa, receberam uma explicação breve, informados sobre a sua natureza e fidelidade. Em seguida receberam um Termo de Consentimento Livre e Esclarecido, e somente aqueles que o assinaram participaram da pesquisa.

Para avaliar e comparar os conhecimentos de diagnóstico dos alunos do antigo e dos alunos do novo currículo de Odontologia da UFSC foi aplicado um questionário, contendo 20 questões objetivas sobre diagnóstico em Odontologia. As questões foram selecionadas do Provão do Sistema Nacional de Avaliação de Ensino Superior dos anos de 2000, 2001, 2002 e 2003.

Após o questionário os alunos responderam também quatro questões objetivas a respeito das suas opiniões em relação ao ponto forte no ensino do curso de Odontologia da UFSC e sobre suas aptidões profissionais.

Os participantes foram divididos em dois grupos, sendo estes chamados de grupo I e grupo II.

O grupo I foi formado por 45 alunos, os quais ingressaram na universidade no segundo semestre de 2006 e colaram grau no final do ano de 2010. Es- 
tes alunos estavam cursando a nona fase e compõem a última turma do currículo antigo de Odontologia da universidade.

O grupo II foi formado por 45 estudantes, os quais ingressaram na universidade no primeiro e segundo semestre de 2007. Estes compõem as duas primeiras turmas do novo currículo, fazendo parte da décima e nona fase respectivamente.

Após a realização do questionário as pontuações foram analisadas estatisticamente através do programa epiData e transportadas para o Excel.

\section{RESULTADOS}

A pesquisa contou com a participação de 90 estudantes, dos quais 24 eram do sexo masculino e 66 do sexo feminino, como pode ser observado na Tabela 1.

Os participantes da pesquisa representaram dois grupos, o grupo I, composto pelos alunos do currículo antigo de odontologia da UFSC e o grupo II, formado pelos alunos do novo currículo de odontologia da UFSC, representados na Tabela 2.

A faixa etária dos alunos variou entre 21 e 43 anos, a média de idade foi de 23,96 com um desvio padrão de 3,092, para melhor compreensão as ida-

Tabela 1 - Participante em relação ao sexo.

\begin{tabular}{|c|c|c|}
\hline Sexo & Frequência & Porcentagem \\
\hline Masculino & 24 & 26,6 \\
\hline Feminino & 66 & 73,4 \\
\hline Total & 90 & 100 \\
\hline
\end{tabular}

Tabela 3 - Média de idade dos participantes.

\begin{tabular}{|c|c|c|c|}
\hline $\begin{array}{c}\text { Idade em } \\
\text { categorias }\end{array}$ & Frequência & Porcentagem & $\begin{array}{c}\text { Média de } \\
\text { idade }\end{array}$ \\
\hline $21-23$ & 58 & 64,4 & - \\
\hline $24-43$ & 32 & 35,6 & - \\
\hline Total & 90 & 100 & 23,96 \\
\hline
\end{tabular}

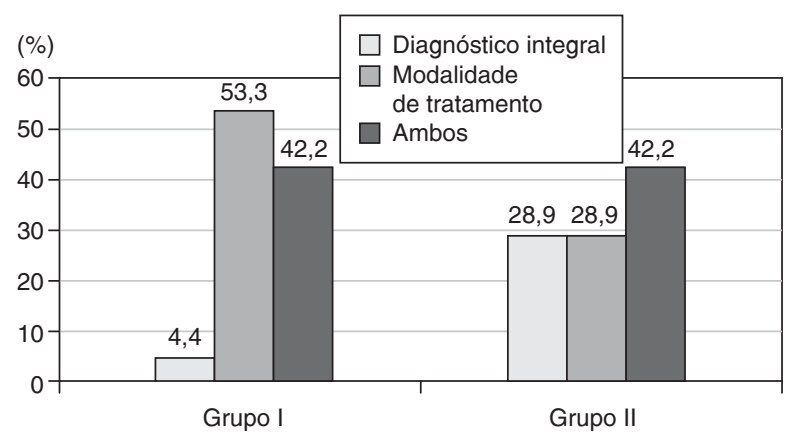

Gráfico 1 - Ponto forte do curso de graduação da UFSC. des foram divididas em categorias como pode ser observado na Tabela 3.

Em relação aos conhecimentos de diagnostico em Odontologia os estudantes do grupo I totalizaram uma média de acertos de 13,57 pontos, número este que corresponde a $67,85 \%$ do total de questões, já os alunos do grupo II obtiveram uma média de 14,11 pontos, que corresponde a $70,55 \%$ do questionário como pode ser observado na Tabela 4 .

Quando questionados sobre o que consideram como ponto forte no ensino do curso de graduação da UFSC 4,4\% dos componentes do grupo I disseram ser o aprendizado em diagnóstico integral, $53,3 \%$ em modalidades de tratamento e $42,2 \%$ em ambos, já no grupo II 28,9\% em diagnóstico integral, $28,9 \%$ em modalidades de tratamento e $42,2 \%$ em ambos conforme o Gráfico 1.

Ao concluir o curso 11,1\% dos estudantes do grupo I disseram que pretendem trabalhar na clínica privada, $28,8 \%$ nos serviços públicos de saúde e $60 \%$ em ambos, no grupo II 6,7\% sentem-se mais capacitados para trabalhar na clínica privada, 35,6\% nos serviços públicos de saúde e 57,8\% em ambos, podemos observar essa relação no Gráfico 2.

Tabela 2 - Total de representantes por grupo.

\begin{tabular}{|c|c|c|}
\hline Grupo & $\mathbf{N}^{\circ}$ de Participantes & Porcentagem \\
\hline Grupo I & 45 & 50 \\
\hline Grupo II & 45 & 50 \\
\hline Total & 90 & 100 \\
\hline
\end{tabular}

Tabela 4 - Média de acertos dos grupos I e II.

\begin{tabular}{|c|c|c|}
\hline Grupos & $\begin{array}{c}\text { Média de acertos } \\
\text { por grupo }\end{array}$ & $\begin{array}{c}\text { Porcentagem total } \\
\text { no questionário }\end{array}$ \\
\hline Grupo I & 13,57 & 67,85 \\
\hline Grupo II & 14,11 & 70,55 \\
\hline
\end{tabular}

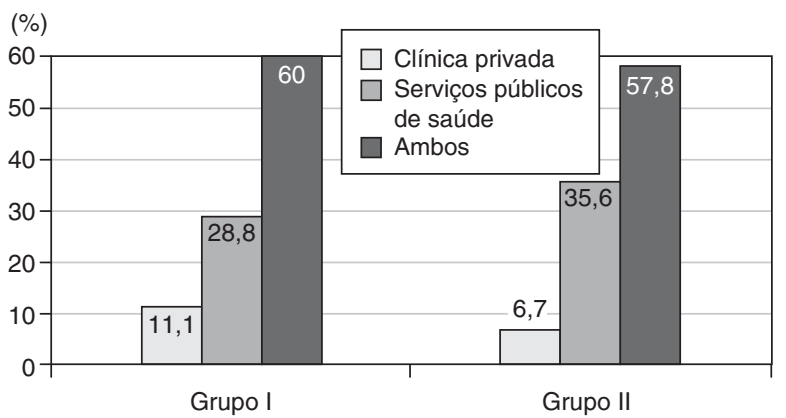

Gráfico 2 - Ao concluir o curso de graduação pretendem trabalhar. 
(\%)

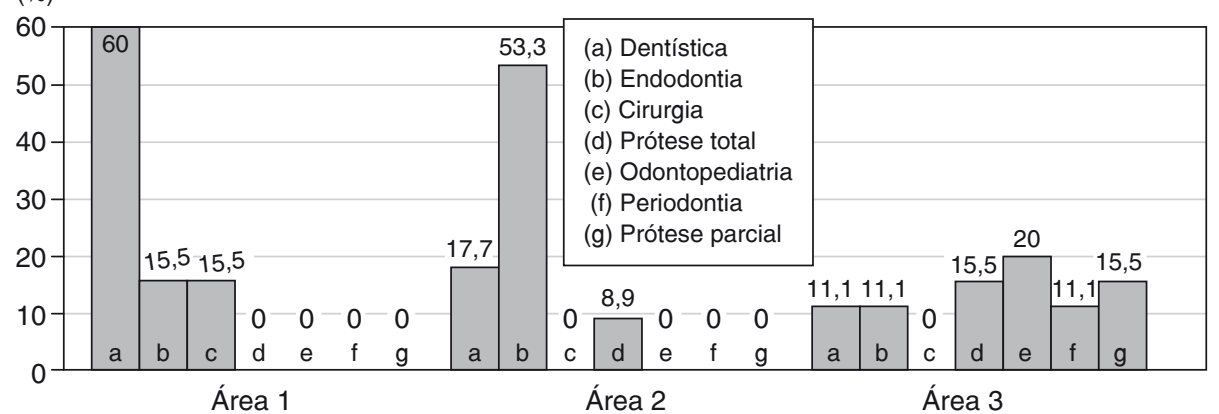

(\%)

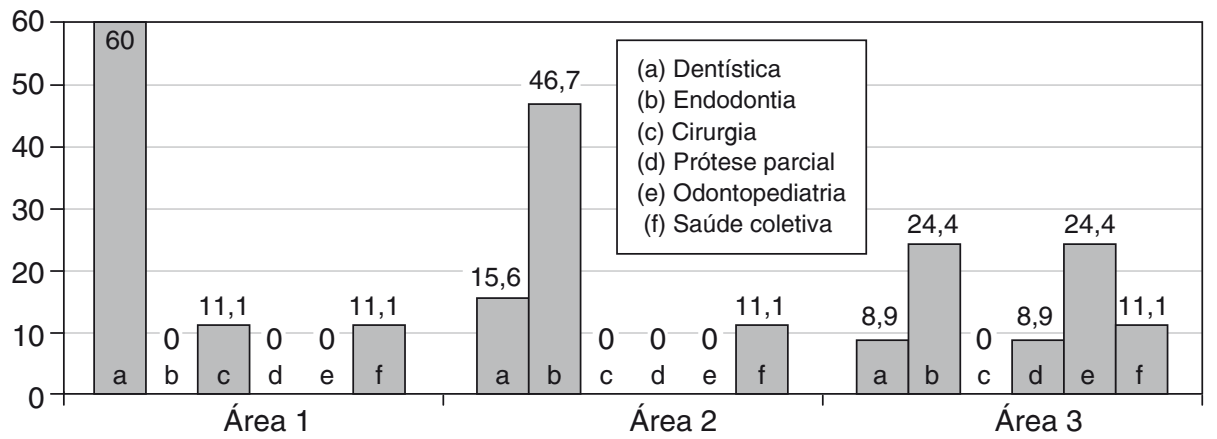

Gráfico 3 - Áreas do conhecimento que o grupo I se sente mais preparado para atuar em ordem crescente.

Gráfico 4 - Áreas do conhecimento que o grupo II se sente mais preparado para atuar em ordem crescente.
Em relação às áreas do conhecimento, os estudantes indicaram as três em ordem crescente que se sentem mais capacitados para atuar. A maioria dos estudantes do grupo I e do grupo II optou pela dentística, seguida pela endodontia e como terceira opção a maioria escolheu a odontopediatria, como pode ser observado nos Gráficos 3 e 4.

\section{DISCUSSÃo}

As necessidades de renovação acompanhadas das intensas mudanças do mundo moderno fizeram com que o currículo do curso de Odontologia da UFSC passasse por transformações (Amante, 2006).

Os problemas relacionados ao modelo dominante de formação na área de Odontologia motivaram discussões no meio acadêmico e profissional visando à necessidade de mudanças no sentido de proporcionar a melhoria da saúde bucal da população brasileira (Abeno, 2002).

No mesmo sentido Morita e Kriger (2006) enfatizam que o próprio conceito do processo saúde-doença precisa ser adequado, com um melhor entendimento da promoção de saúde, da prevenção e do controle das doenças, dos meios de diagnóstico e de tratamento e, principalmente, da manutenção da saúde.

Neste estudo foram avaliados os conhecimentos de diagnóstico dos alunos do currículo antigo (grupo I) e dos alunos do currículo novo (grupo II) de
Odontologia da Universidade Federal de Santa Catarina, por meio de um questionário contendo vinte questões objetivas.

O presente estudo demonstra que o grupo I obteve uma média de acertos de 13,57 pontos que corresponde a $67,85 \%$ do questionário, já o grupo II obteve uma média de 14,11 pontos valor este que corresponde a $70,55 \%$, o que nos remete a pensar que mesmo o grupo II obtendo uma maior porcentagem de acertos (Tabela 4), esta não foi estatisticamente significativa em relação ao grupo I, ainda que os grupos não tenham apresentado diferença estatisticamente significativa em seus resultados ambos obtiveram uma media relevante de acertos que vai de encontro com a afirmação de Rodrigues e Serpa (2001) que dizem que a Odontologia vem intensificando investigações e estudos que permitem ao profissional a mais adequada compreensão do paciente e das suas circunstâncias. A humanidade ingressou numa era onde a interação entre as ciências projetou o aprimoramento da vida. Este processo exige um conhecimento mais amplo e seguro, de relações psicossociais e intra/interpessoais de forma a relacioná-las com o quadro clínico apresentado pelo paciente. No entanto Morita e Kriger (2004) lembram que no campo da Odontologia os movimentos de mudança dos modelos tradicionais de formação profissional são mais recentes em relação às outras áreas da saúde. Reforçando os dados 
desta pesquisa a compreensão do paciente e das suas circunstâncias segundo Matos (2006) é imprescindível para favorecer a execução de procedimentos odontológicos, auxiliando diagnósticos de doenças bucais e favorecendo o processo educativo no sentido de manter-se em saúde, talvez refletindo sobre estas questões não seja possível conceber as técnicas odontológicas apenas com uma função instrumental e como meio de subsistência, mas também como meio de trazer qualidade de vida para as pessoas; se incorporarmos a ela valores humanos. Neste sentido Feuerwerker e Sena (1999) afirmam que as mudanças no campo da Odontologia são resultado de elementos como as novas modalidades de organização do mundo do trabalho em saúde. Segundo as Diretrizes da Política Nacional de Saúde Bucal as ações de saúde bucal devem se inserir na estratégia planejada pela equipe de saúde numa inter-relação permanente com as demais ações da Unidade de Saúde (Brasil, 2004).

Neste estudo também se verificou a opinião dos estudantes em relação ao ponto forte de ensino do curso de Odontologia da UFSC (Gráfico 1), o qual apresentou diferença estatisticamente significativa. Os alunos do grupo I apontaram em sua maioria o aprendizado em modalidades de tratamento $53,3 \%$, já no grupo II essa alternativa foi assinalada por apenas $28,9 \%$. Dos 45 estudantes do grupo I somente $4,4 \%$ disseram ser o aprendizado em diagnóstico integral, em contrapartida esta alternativa foi assinalada por $28,9 \%$ dos estudantes do grupo II e $42,2 \%$ tanto do grupo I como do grupo II responderam que o ponto forte do curso é tanto o aprendizado em diagnóstico integral como em modalidades de tratamento. Desta forma podemos observar uma maior prevalência no aprendizado em diagnóstico integral no grupo II, resultado este de grande relevância para esta pesquisa, sobretudo por esta ter como objetivo avaliar o novo currículo de odontologia da UFSC, neste sentido Buss (2000) salienta que na atenção integral evidencia-se como uma ponte que liga as demais áreas do conhecimento. Para Pinheiro e Luz (2003) aceitar a integralidade como objetivo norteador de novas formas de agir em saúde e, por que não, de uma nova forma de gestão de cuidados nas instituições de saúde, proporcionando o surgimento de novas experiências e desenvolvimento de novas tecnologias assistenciais. Gondim (2002) relembra que em um curso da área da saúde, observa-se a relação de integração, onde o paciente é visto como um todo e, não simples- mente como uma parte. Feuerwerker (2002) aponta que a possibilidade de atenção integral implica num aumento dos referenciais com que cada profissional de saúde trabalha na estruturação de seu repertório de compreensão e ação. Em paralelo, o reconhecimento da limitação da ação uniprofissional para dar conta das necessidades de saúde de indivíduos e populações. Ressalta que a atenção integral exige mudanças nas relações de poder entre profissionais de saúde (para que efetivamente constituam uma equipe multiprofissional) e entre profissionais de saúde e usuários (para que se amplie efetivamente sua autonomia).

Quando questionados sobre onde gostariam de trabalhar ao concluir o curso (Gráfico 2), os resultados são semelhantes em ambos os grupos, 11,1\% dos estudantes do grupo I e 6,7\% do grupo II disseram ser na clínica privada, $28,9 \%$ do grupo I e $35,5 \%$ do grupo II responderam ser em serviços públicos de saúde, já aproximadamente $60 \%$ dos alunos de ambos os grupos responderam tanto na clínica privada como em serviços públicos de saúde. De acordo com os resultados do trabalho de Matos (2006) quando os alunos são questionados acerca dos seus planos profissionais ao se expressarem mais especificamente, os planos que mais se destacam são: o desejo de fazer especialização/ mestrado/doutorado $(26,8 \%)$ e ir para o serviço público $(25,5 \%)$ e, em menor proporção, trabalhar como autônomo $(11,2 \%)$ e em clínicas privadas, terceirizando a sua mão de obra $(8,5 \%)$. Do mesmo modo no trabalho realizado por Unfer (2004) apenas uma minoria pretende exercer exclusivamente a clínica particular. Neste sentido Koide et al. (2004) afirmam que os recém-formados têm receio em abrir a sua própria clínica e estão incertos se conseguirão arcar com todos os encargos e despesas; em sua pesquisa $50 \%$ dos profissionais que tinham até dois anos de formados exerciam suas atividades clínicas como empregados de outros profissionais ou instituição. Para Chaves (2005), as dificuldades de ingresso no mercado privado têm evidenciado o trabalho no setor público como uma boa alternativa.

Procurou-se neste estudo identificar quais áreas do conhecimento os estudantes apontam como as que se sentem mais capacitados para atuar ao se formar (Gráficos 3 e 4). Os resultados tanto do grupo I como do grupo II foram muito semelhantes, no grupo I as áreas mais indicadas pelos estudantes foram dentística, endodontia, cirurgia, pró- 
tese total, odontopediatria e periodontia, sendo a dentística, seguida pela endodontia e odontopediatria $(60 \%, 53,3 \%$ e $20 \%$ respectivamente) aquelas com maior prevalência. No grupo II as áreas mais indicadas foram as mesmas do grupo I exceto a periodontia, já que no grupo II predominou a saúde coletiva $(60 \%, 46,7 \%$ e $24,4 \%$ para dentística, endodontia e odontopediatria respectivamente). Observou-se que a saúde coletiva não foi citada pelo grupo I, no entanto no grupo II ela manteve a mesma popularidade: $11,1 \%$ quando citada como opção 1, 2 ou 3. Concordando com estes resultados, Matos (2006) observou em sua pesquisa elevado interesse dos alunos por disciplinas e temas da área Clínica $(74,8 \%$ e $74,4 \%$, respectivamente) e o baixo nível de interesse pelas disciplinas e temas das áreas de ciências humanas $(0,8 \%)$ e saúde coletiva $(8,4 \%)$. O autor ressalta que esses resultados, refletem a própria organização curricular dos cursos, uma vez que estes apresentam uma maior proporção de disciplinas clínicas. Esta condição parece necessária para uma boa formação técnica dos alunos e também refletem que o currículo oculto vai guiando os alunos durante quase todo o processo de formação. $\mathrm{O}$ valor que se atribui à formação competente está fundamentado no componente técnico-científico e todo conhecimento ou prática que esteja inserida fora desse contexto é considerado aprendizado marginal. Embora não apareça de forma amplamente significativa os interesses por saúde coletiva estão crescendo entre os estudantes de Odontologia. Interessante é que este critério somente se manifestou no grupo II, ou seja, nos estudantes do novo currículo. Diante desde fato Silva (2010) lembra que é importante tratar assuntos dentro do contexto social, formando profissionais com capacidade para atuar em todos os níveis de atenção à saúde e não exclusivamente para atuar numa prática clínica, sem considerar os aspectos demográficos e suas consequências epidemiológicas.

Esse fazer e refazer revela o caráter processual e dinâmico da elaboração de uma reorganização curricular e a existência, por trás dele, de um processo de avaliação permanente do que já se consagrou como prática e fazer docente, a fim de legitimar o que permanece e o que muda no futuro. A avaliação de um currículo em vigor inaugura o processo de reorganização curricular, mas não se esgota nesse momento inicial. Em outras palavras, todas as decisões subsequentes à avaliação inicial, a avalia- ção diagnóstica, dependem dela, mas ao mesmo tempo a retomam e a reconstroem (Ribeiro; Silva, 2007). Como discute Cappelletti (2002), currículo é um processo abrangente, complexo e dinâmico que ultrapassa as grades curriculares e envolve pessoas e suas relações durante a participação no processo educativo.

A análise neste estudo sugere que o preparo do aluno do curso de Odontologia da UFSC, nos aspectos relacionados à Reforma Curricular está passando por transformações de forma gradual, ou seja, está em transição. Sabe-se que um processo de mudança envolvendo pessoas não é efetuado de forma rápida e completa. Necessita de avaliações frequentes e permanentes. Portanto, este trabalho contribui para a reflexão deste processo.

Quanto à limitação deste estudo podemos destacar o fato da Reforma Curricular ser uma questão ainda recente e em construção no curso, por ter sido observado somente por três aspectos da mesma, seria interessante que novos estudos fossem realizados de forma mais abrangente envolvendo, alunos, docentes e demais contribuintes do processo. Por outro lado, a maior contribuição deste estudo é que mesmo não obtendo valores de extrema significância observou-se que alguns aspectos da reforma curricular como o aprendizado em diagnóstico integral vem fazendo parte do dia a dia dos futuros profissionais que participaram desta pesquisa.

\section{CONCLUSÃo}

Com base nos métodos empregados e nos resultados obtidos, conclui-se que:

- Em relação aos conhecimentos de diagnóstico em Odontologia ambos os grupos apresentaram resultados semelhantes com uma média de aproximadamente setenta por cento de acertos.

- O curso de Odontologia da UFSC tem seu foco de ensino direcionado para o aprendizado diagnóstico integral.

- A maior parte dos estudantes pretende trabalhar nos serviços públicos de saúde concomitantemente com a clínica privada.

- Tanto os estudantes do grupo I como do grupo II apontam as disciplinas clínicas em especial a dentística, endodontia e odontopediatria, como aquelas que se sentem mais preparados para atuar. Não obstante a este fato a saúde coletiva vem se destacando no grupo II como uma tendência para esta nova geração de profissionais. 


\section{ABSTRACT}

\section{Evaluation of the curriculum reform of the University of Santa Catarina dentistry course}

The dentistry course of the Federal University of Santa Catarina has recently undergone curricular changes based on the National Curriculum Guidelines for undergraduate courses in dentistry. The change in the university's educational project was implemented in 2006, in accordance with Resolution \#3/02 CNE/CES, dated February 19, 2002, which established the National Curriculum Guidelines for Undergraduate Dentistry Courses. The new curriculum came into force in the first half of 2007. With this in mind, the main goal of this study was to evaluate, on one hand, the curriculum reform, by comparing the diagnostic knowledge of students of the old and of the new curriculum, and, on the other hand, the views of these students on the course and their professional aptitudes. The survey was conducted by applying a questionnaire containing 20 questions about diagnoses made in dentistry and 4 undergraduate course-related questions about the student's professional aptitudes. The study included 90 students who were separated into two groups: Group I was formed by students of the old curriculum, and Group II was formed by students of the new curriculum. After the questionnaires were administered, the data were analyzed statistically in the epiData program and entered in Excel. The results showed that the differences in diagnostic knowledge between groups I and II were not statistically significant. In relation to the strong point of the course, there was a statistical difference between the two groups. What prevailed in Group I was the learning of treatment modalities, whereas, in Group II, it was the learning of complete diagnoses. In relation to where the students would prefer to work, the majority of both groups responded that they preferred public health services together with private clinic practice. In respect to the areas in which they preferred to work, both groups opted mostly for clinical disciplines, in that group II had a significant preference for Collective Health.

\section{DESCRIPTORS}

Diagnosis. Interdisciplinarity. Dentistry. Curriculum. Curriculum Guidelines.

\section{REFERÊNCIAS}

1. Almeida, M. Diretrizes curriculares nacionais para os cursos universitários da área da saúde. Rede Unida, Londrina, 2003.
2. Amante, J.C. Projeto político pedagógico do curso de graduação em odontologia da Universidade Federal de Santa Catarina. Florianópolis, 2006.

3. Associação Brasileira do Ensino Odontológico. Evolução dos Cursos. Disponível em: < http://www.abeno.org.br/ > . Acesso em 10 de setembro de 2010.

4. Associação Brasileira do Ensino Odontológico. Análise sobre as diretrizes curriculares nacionais para os cursos de graduação em odontologia. Rev Abeno, v.1, n.3, p.35-8, 2002.

5. Ayres, J.R.C.M. O cuidado, os modos de ser (do) humano e as práticas de saúde. Saúde e Sociedade, Rio de Janeiro, v.13, n.3, p.16-29, 2004.

6. Badan, D.E.C., Marcelo, V.C., Rocha, D.G. Percepção e utilização dos conteúdos de saúde coletiva por cirurgiões-dentistas egressos da Universidade Federal de Goiás. Ciênc. Saúde Coletiva, Rio de Janeiro, v.15, 2010.

7. Bitencourt, E. A Odontologia de 1990 até nossos dias. Florianópolis, 1994.

8. Brasil. Constituição da República Federativa do Brasil. Brasília: Senado Federal, 1988.

9. Brasil. Lei no 9.394. Estabelece as diretrizes e bases da educação nacional. Diário Oficial da União, 1996.

10. Brasil. Ministério da Educação. Resolução no 3/02 CNE/ CES, de 19 de fevereiro de 2002, que institui as Diretrizes Curriculares Nacionais do Curso de Graduação em Odontologia. Disponível em: < http://portal.mec.gov.br/sesu/arquivos/ pdf $/ 0302^{\circ}$ dontologia.pdf $>$. Acesso em: 10 de setembro de 2010.

11. Brasil. Ministério da Saúde. Lei orgânica da saúde. Brasília, Ministério da Saúde, 1996. Promoção da saúde: Cartas de Otawa, Declaração de Adelaide, Sundsvall e Santa Fé de Bogotá. Tradução de L.E. Fonseca. Brasília, 1996.

12. Brasil. Ministério da Saúde. Caderno de atenção básica nº17. Saúde Bucal. Brasília, 2006.

13. Brasil. Ministério da Saúde. Diretrizes da Política Nacional de Saúde Bucal. Brasília, 2004.

14. Buss, P.M. Promoção da saúde e qualidade de vida. Ciência \& Saúde Coletiva, Rio de Janeiro, v. 5, n. 1, p. 163-177, 2000.

15. Cappelletti, I.F. Avaliação de currículos e possibilidades. In: Avaliação de Políticas e práticas educacionais (Cappelletti, I.F.ORG.), São Paulo, Editora Articulção Universidade/Escola Ltda, 2002.

16. Ceccim, R.B. Criança hospitalizada: a atenção integral como uma escuta à vida. In: Ceccim R. B, Carvalho P. R. A, organizadores. Criança hospitalizada: atenção integral como escuta à vida. Porto Alegre: Editora da Universidade, p. 27-41, 1997.

17. Ceccim, R.B. Educação Permanente em Saúde: descentralização e disseminação de capacidade pedagógica na saúde. Ciênc. saúde coletiva, Rio de Janeiro, v.10, n.4, 2005.

18. Ceccim, R.B., Feuerwerker, L.C.M. Mudança na graduação das profissões de saúde sob o eixo da integralidade. Cad. Saú- 
de Pública, Rio de Janeiro, v. 20, n. 5, p. 1400-1410, 2004a.

19. Ceccim, R.B., Feuerwerker, L.C.M. O quadrilátero da formação para a área da saúde: ensino, gestão, atenção e controle social. Physis, Rio de Janeiro, v. 14, n. 1, p. 41-65, 2004 b.

20. Chaves, S.C.L. A atenção à saúde bucal, a descentralização e o espaço social. Tese (Doutorado em Saúde Coletiva) - Instituto de Saúde Coletiva, UFBA, Salvador, 2005.

21. Costa, I.C.C. Os sete saberes necessários à educação do futuro e o planejamento das ações de saúde: algumas reflexões e confluências. Rev da Abeno, v. 7, n.2, p.122-129, 2007.

22. Costa, I.C.C., Araújo, M.N.T. Definição do perfil de competências em saúde coletiva a partir da experiência de cirurgiões-dentistas atuantes no serviço público. Ciênc. Saúde Coletiva, Rio de Janeiro, v.16, n.1, 2011.

23. Deslandes, S.F. Análise do discurso oficial sobre a humanização da assistência hospitalar. Ciência e Saúde Coletiva, v. 9, n.1, p. 7-14, 2004.

24. Feuerwerker, L.C.M., Sena, R.R. A construção de novos modelos acadêmicos, de atenção à saúde e de participação social. In: Feuerwerker L, Almeida M, Llanos, C.M. A educação dos profissionais de saúde na América Latina: teoria e prática de um movimento de mudança. Tomo 1 - Um olhar analítico. São Paulo: Editora Hucitec/ Buenos Aires: Lugar Editorial/ Londrina: Editora UEL, p. 47-81, 1999.

25. Feuerwerker, L.C.M. Estratégias para a mudança da formação dos profissionais de saúde. Caderno de Currículo e Ensino, n. 2, p. 11-23, 2001.

26. Feuerwerker, L.C.M. Além do discurso de mudança da Educação Médica: processos e resultados. São Paulo: Hucitec, 2002.

27. Feuerwerker, L.C.M. Reflexões sobre as experiências de mudança na formação dos profissionais de saúde. Olho Mágico, n. 10, p. 21-6, 2003.

28. Feuerwerker, L.C.M., Cecílio, L.C.O. O hospital e a formação em saúde: desafios atuais. Ciênc. saúde coletiva, Rio de Janeiro, v.12, n.4, 2007.

29. Gondim, S.M.G. Perfil profissional e mercado de trabalho: relação com a formação acadêmica pela perspectiva de estudantes universitários. Estudos de psicologia, v.7, n.2, p.299 309, 2002.

30. Hernández, D. Políticas de certificación de competências em América Latina. Boletín Técnico Interamericano de Formación Profesional. Montevideo, n.152, 2002.

31. Koide, E.R., Paranhos, L.R., Quintela, R.S. Análise do perfil profissional na odontologia. Revista Paulista de Odontologia. Ano XXVI, n. 3, p. 17- 22, 2004.

32. Lima, V.V. Competência: distintas abordagens e implicações na formação de profissionais de saúde. Interface, Botucatu, v. 9, n. 17, p. 369-379, 2005.

33. Maltagliati, L.A., Goldenberg, P. Reforma curricular e pesquisa na graduação em odontologia: uma história em construção. Hist. Ciênc. Saúde, Rio de Janeiro, v. 14, n. 4, 2007.
34. Martins, C.B. O ensino superior brasileiro nos anos 90. São Paulo Perspec., São Paulo, v. 14, n. 1, p. 41-60, 2000.

35. Matos, M.S de. Análise do perfil dos alunos e da dimensão éticohumanística na formação de cirurgiões-dentistas em dois cursos de Odontologia da Bahia. Tese de Pós-graduação em Educação. Salvador, 2006.

36. Melo, E.M. Ação comunicativa, democracia e saúde. Ciênc. Saúde Coletiva, Rio de Janeiro, v. 10, p.167-178, 2005.

37. Minayo, M.C.S. O desafio do conhecimento: pesquisa qualitativa em saúde. $7^{\mathrm{a}}$ edição, São Paulo/Rio de Janeiro: Hucitec-Abrasco, 2000.

38. Morin, E., et al. Educação e complexidade: os sete saberes e outros ensaios. 3. ed. São Paulo: Cortez, 2005.

39. Morita, M.C, Kriger, L. Mudanças nos cursos de Odontologia e a interação com o SUS. Revista da ABENO, Taguatinga/DF, v. 4, n. 1, p. 17-21, 2004.

40. Morita M.C, Kriger L. A relação ensino e serviços de odontologia. In: Carvalho A.C.P, Kriger L. Educação odontológica. São Paulo: Artes Médicas, p. 129-39, 2006.

41. Nóvoa, A. Os professores e sua formação. Lisboa: D. Quixote, p. 15-34, 1992.

42. Petroianu A. Ética, moral e deontologia médicas. Rio de Janeiro: Guanabara Koogan, 2000.

43. Pinheiro, R., Luz, M.T. Práticas eficazes x modelos ideais: ação e pensamento na construção da integralidade. In: Pinheiro, R.; Mattos, R. A. (Org.). Construção da integralidade: cotidiano, saberes e práticas em saúde. Rio de Janeiro, IMS-UERJ, p. 7-34, 2003.

44. Rede unida. Sobre a proposta de diretrizes curriculares para a medicina. Olho Mágico, n. 5, p. 11-3, 1999.

45. Ribeiro, E.C.O. Representações de alunos e docentes sobre as práticas de cuidado e de formação: uma avaliação de experiências de mudança em escolas médicas. Tese (Doutorado) Instituto de Medicina Social, Universidade do Estado do Rio de Janeiro, Rio de Janeiro, 2003.

46. Ribeiro, M.P O., Silva, M.C.V.M. Análise de um processo de reformulação curricular no ensino superior. Revista E-Curriculum, São Paulo, v. 3, n. 1, 2007.

47. Rodrigues, N. Educação: da formação humana à construção do sujeito ético. Educ. Soc., Campinas, v. 22, n. 76, p. 232-257, 2001.

48. Rodrigues, R., Serpa, A.R. Perfil bioemocional do paciente e o controle de placa bacteriana. p. 75-85. In: Oppermann, Rui; Rösing, Cassiano (Org.). Periodontia: ciência e clínica. Artes Médicas, 2001.

49. Rosa, J.E. A Faculdade de Odontologia da Rua São Francisco. Florianópolis, 1994.

50. Rosa, J.E., Madeira, A.A. Odontologia Catarinense. Evolução, ensino e movimento associativo. Ed. da UFSC, Florianópolis, p. 79-240, 1982.

51. Santos, L.A.S., et al. Projeto pedagógico do programa de gra- 
duação em nutrição da Escola de Nutrição da Universidade Federal da Bahia: uma proposta em construção. Rev. Nutr., Campinas, v. 18, n. 1, p. 105-117, 2005.

52. Santos, J.L.F., Westphal, M.F. Práticas emergentes de um novo paradigma de saúde: o papel da universidade. Estud. av., São Paulo, v. 13, n. 35, p.71-88, 1999.

53. Silva, C.P.C. da. Competências e habilidade requeridas na formação do cirurgião dentista: percepção de professores e alunos. Santa Maria, RS, 2010.

54. Sousa, C.N. Colaborador sobre os relatos históricos do curso de Odontologia da Universidade Federal de Santa Catarina. Professor da disciplina de Dentística da Universidade Federal de Santa Catarina. Florianópolis, 2010.
55. Sung J.M., Silva J.C. Conversando sobre ética e sociedade. $13^{\mathrm{a}}$ ed. Rio de Janeiro: Vozes, 1995.

56. Unfer, B. et al. Expectativas dos acadêmicos de Odontologia quanto a formação e futura profissão. Saúde, Santa Maria, RS, v.30, n.1-2, p.33-40, 2004.

57. Westphal M.F., Mendes R. Cidade Saudável: uma experiência de Interdisciplinaridade e intersetorialidade. Revista de Administração Pública - RAP - Rio de Janeiro, FGV, v. 34, n. 6, p. 47-61, 2000.

Recebido em $08 / 10 / 2012$

Aceito em 10/12/2012 